\title{
The genome sequence of the seven-spotted ladybird,
}

\section{Coccinella septempunctata Linnaeus, 1758 [version 1; peer}

\section{review: 2 approved]}

\author{
Liam M. Crowley (iD1,
}

University of Oxford and Wytham Woods Genome Acquisition Lab,

Darwin Tree of Life Barcoding collective,

Wellcome Sanger Institute Tree of Life programme,

Wellcome Sanger Institute Scientific Operations: DNA Pipelines collective, Tree of Life Core Informatics collective, Darwin Tree of Life Consortium

${ }^{1}$ Department of Zoology, University of Oxford, Oxford, UK

V1 First published: 24 Nov 2021, 6:319

https://doi.org/10.12688/wellcomeopenres.17346.1

Latest published: 24 Nov 2021, 6:319

https://doi.org/10.12688/wellcomeopenres.17346.1

\section{Abstract}

We present a genome assembly from an individual female Coccinella septempunctata (the seven-spotted ladybird; Arthropoda; Insecta; Coleoptera; Coccinellidae). The genome sequence is 399 megabases in span. The majority (99.96\%) of the assembly is scaffolded into 9 chromosomal pseudomolecules, with the $\mathrm{X}$ sex chromosome assembled.

Keywords

Coccinella septempunctata, seven-spotted ladybird, genome sequence, chromosomal

This article is included in the Tree of Life gateway.

\section{Open Peer Review \\ Approval Status \\ 1 \\ 2 \\ version 1 \\ 24 Nov 2021

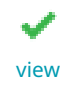 \\ view
1. Stephen Richards , Baylor College of Medicine, Houston, USA
2. Jongsun Park ID, InfoBoss Inc, Seoul, South Korea

Any reports and responses or comments on the article can be found at the end of the article. 
Corresponding author: Darwin Tree of Life Consortium (mark.blaxter@sanger.ac.uk)

Author roles: Crowley LM: Investigation, Resources, Writing - Original Draft Preparation, Writing - Review \& Editing;

Competing interests: No competing interests were disclosed.

Grant information: This work was supported by the Wellcome Trust through core funding to the Wellcome Sanger Institute (206194) and the Darwin Tree of Life Discretionary Award (218328).

The funders had no role in study design, data collection and analysis, decision to publish, or preparation of the manuscript.

Copyright: (c) 2021 Crowley LM et al. This is an open access article distributed under the terms of the Creative Commons Attribution License, which permits unrestricted use, distribution, and reproduction in any medium, provided the original work is properly cited.

How to cite this article: Crowley LM, University of Oxford and Wytham Woods Genome Acquisition Lab, Darwin Tree of Life Barcoding collective et al. The genome sequence of the seven-spotted ladybird, Coccinella septempunctata Linnaeus, 1758 [version 1; peer review: 2 approved] Wellcome Open Research 2021, 6:319 https://doi.org/10.12688/wellcomeopenres.17346.1

First published: 24 Nov 2021, 6:319 https://doi.org/10.12688/wellcomeopenres.17346.1 


\section{Species taxonomy}

Eukaryota; Metazoa; Ecdysozoa; Arthropoda; Hexapoda; Insecta; Pterygota; Neoptera; Endopterygota; Coleoptera; Polyphaga; Cucujiformia; Coccinellidae; Coccinellinae; Coccinellini; Coccinella; Coccinella septempunctata Linnaeus, 1758 (NCBI:txid41139).

\section{Background}

The 7-spot ladybird, Coccinella septempunctata Linnaeus, 1758, is an iconic species of ladybird and one of the most common in the UK and across Europe. It is widespread and abundant throughout its native range of Europe, Asia and North Africa, although it's distribution trend in the UK is decreasing (Roy \& Brown, 2018). It can be found across a wide range of habitats including gardens and agricultural land. Adults are large (5-8 mm), conspicuously marked species with vivid red elytra marked with 7 black spots. The head, pronotum and legs are black. The scarce 7-spot ladybird, Coccinella magnifica, is very similar, but can be distinguished by its larger black spots, and additional pair of white markings below the legs on the underside. The 7-spot ladybird is a predatory species, feeding on a wide range of aphid species both as a larva and as an adult. It overwinters as an adult in among foliage, dead plant material and leaf litter. The broad geographic success of this species may be underpinned by its ecological plasticity based on both genetic and phenotypic polymorphisms (Hodek et al., 2013). It has been repeatedly introduced to North America as a biological control agent against aphids in agricultural systems.

\section{Genome sequence report}

The genome was sequenced from one female $C$. septempunctata (Figure 1) collected from Wytham Farm, Oxfordshire (biological vice-county: Berkshire), UK (latitude 51.779, longitude -0.317). A total of 76-fold coverage in Pacific Biosciences singlemolecule long reads and 89-fold coverage in 10X Genomics read clouds were generated. Primary assembly contigs were scaffolded with chromosome conformation Hi-C data. Manual assembly curation corrected 78 missing/misjoins and removed 10 haplotypic duplications, reducing the assembly length by $2.60 \%$ and the scaffold number by $70.37 \%$, and increasing the scaffold N50 by $49.73 \%$.

The final assembly has a total length of $399 \mathrm{Mb}$ in 24 sequence scaffolds with a scaffold N50 of $41.4 \mathrm{Mb}$ (Table 1). The majority,

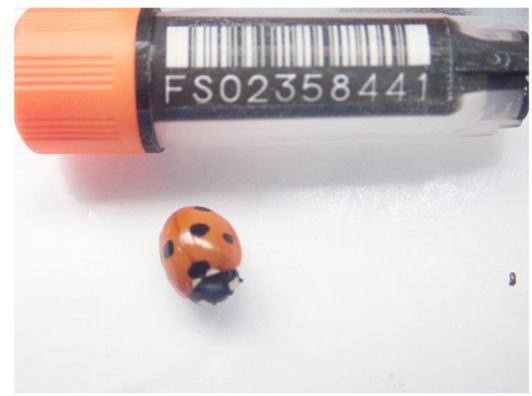

Figure 1. Image of the icCocSept1 specimen captured prior to preservation and processing.
$99.96 \%$, of the assembly sequence was assigned to 10 chromosomal-level scaffolds, representing 9 autosomes (numbered by sequence length), and the $\mathrm{X}$ sex chromosome (Figure 2Figure 5; Table 2). There is a repeat that is shared between chromosomes $\mathrm{X}$ and 9 that could be slightly differently distributed between the two. The assembly has a BUSCO v5.1.2 (Manni et al., 2021) completeness of $97.5 \%$ (single $96.4 \%$, duplicated $1.0 \%$ ) using the endopterygota_odb10 reference set. While not fully phased, the assembly deposited is of one haplotype. Contigs corresponding to the second haplotype have also been deposited.

\section{Methods}

Sample acquisition and nucleic acid extraction

A single female C. septempunctata was collected from Wytham Farm, Oxfordshire (biological vice-county: Berkshire), UK

Table 1. Genome data for Coccinella septempunctata, icCocSept1.1.

\begin{tabular}{|c|c|}
\hline \multicolumn{2}{|l|}{ Project accession data } \\
\hline Assembly identifier & icCocSept1.1 \\
\hline Species & Coccinella septempunctata \\
\hline Specimen & icCocSept1 \\
\hline NCBI taxonomy ID & NCBI:txid41139 \\
\hline BioProject & PRJEB44834 \\
\hline BioSample ID & SAMEA7520205 \\
\hline Isolate information & Female, whole organism \\
\hline \multicolumn{2}{|l|}{ Raw data accessions } \\
\hline PacificBiosciences SEQUEL II & ERR6436372, ERR6558185 \\
\hline 10X Genomics Illumina & ERR6054712-ERR6054715 \\
\hline Hi-C Illumina & ERR6054716 \\
\hline \multicolumn{2}{|l|}{ Genome assembly } \\
\hline Assembly accession & GCA_907165205.1 \\
\hline $\begin{array}{l}\text { Accession of alternate } \\
\text { haplotype }\end{array}$ & GCA_907165185.1 \\
\hline Span (Mb) & 399 \\
\hline Number of contigs & 110 \\
\hline Contig N50 length (Mb) & 16.5 \\
\hline Number of scaffolds & 24 \\
\hline Scaffold N50 length (Mb) & 41.4 \\
\hline Longest scaffold (Mb) & 71.2 \\
\hline BUSCO* genome score & $\begin{array}{l}\text { C:97.5\%[S:96.4\%,D:1.0\%],F: } \\
\text { 1.0\%,M:1.5\%,n:2124 }\end{array}$ \\
\hline \multicolumn{2}{|c|}{$\begin{array}{l}\text { *BUSCO scores based on the endopterygota_odb10 BUSCO } \\
\text { set using v5.1.2. C= complete [S= single copy, D=duplicated], } \\
\text { F=fragmented, M=missing, n=number of orthologues in comparison } \\
\text { A full set of BUSCO scores is available at https://blobtoolkit. } \\
\text { genomehubs.org/view/icCocSept1.1/dataset/CAIRAZ01/busco. }\end{array}$} \\
\hline
\end{tabular}




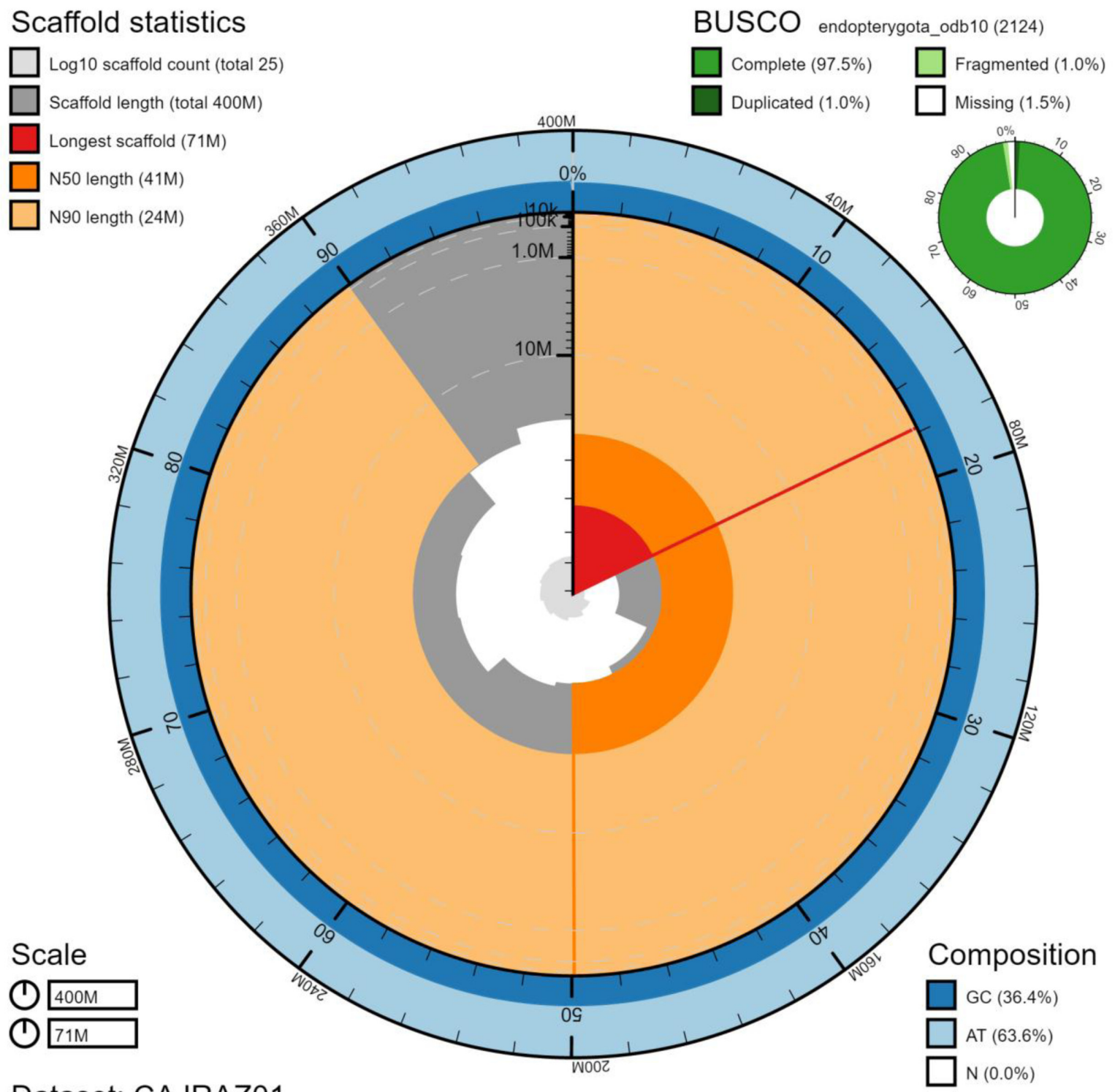

\section{Dataset: CAJRAZ01}

Figure 2. Genome assembly of Coccinella septempunctata, icCocSept1.1: metrics. The main plot is divided into 1,000 size-ordered bins around the circumference with each bin representing $0.1 \%$ of the $398,868,586 \mathrm{bp}$ assembly. The distribution of scaffold lengths is shown in dark grey with the plot radius scaled to the longest scaffold present in the assembly $(71,177,040 \mathrm{bp}$, shown in red). Orange and pale-orange arcs show the N50 and N90 scaffold lengths (41,442,133 and 24,000,787 bp), respectively. The pale grey spiral shows the cumulative scaffold count on a log scale with white scale lines showing successive orders of magnitude. The blue and pale-blue area around the outside of the plot shows the distribution of GC, AT and N percentages in the same bins as the inner plot. A summary of complete, fragmented, duplicated and missing BUSCO genes in the endopterygota_odb10 set is shown in the top right. An interactive version of this figure is available at https://blobtoolkit.genomehubs.org/view/icCocSept1.1/dataset/CAJRAZ01/snail.

(latitude 51.779, longitude -0.317) by Liam Crowley, University of Oxford, using a pooter. The sample was identified by the same individual and snap-frozen on dry ice.

DNA was extracted from the whole organism of icOcyOlen1 at the Wellcome Sanger Institute (WSI) Scientific Operations core from the whole organism using the Qiagen MagAttract HMW DNA kit, according to the manufacturer's instructions. Following this, further DNA was extracted for a PacBio top-up.
Tissue was cryogenically disrupted to a fine powder using a Covaris cryoPREP Automated Dry Pulveriser, receiving multiple impacts. Fragment size analysis of 0.01-0.5 ng of DNA was then performed using an Agilent FemtoPulse. High molecular weight (HMW) DNA was again extracted using the Qiagen MagAttract HMW DNA extraction kit. HMW DNA was sheared into an average fragment size between $12-20 \mathrm{~kb}$ in a Megaruptor 3 system with speed setting 30. Sheared DNA was purified by solid-phase reversible immobilisation using 


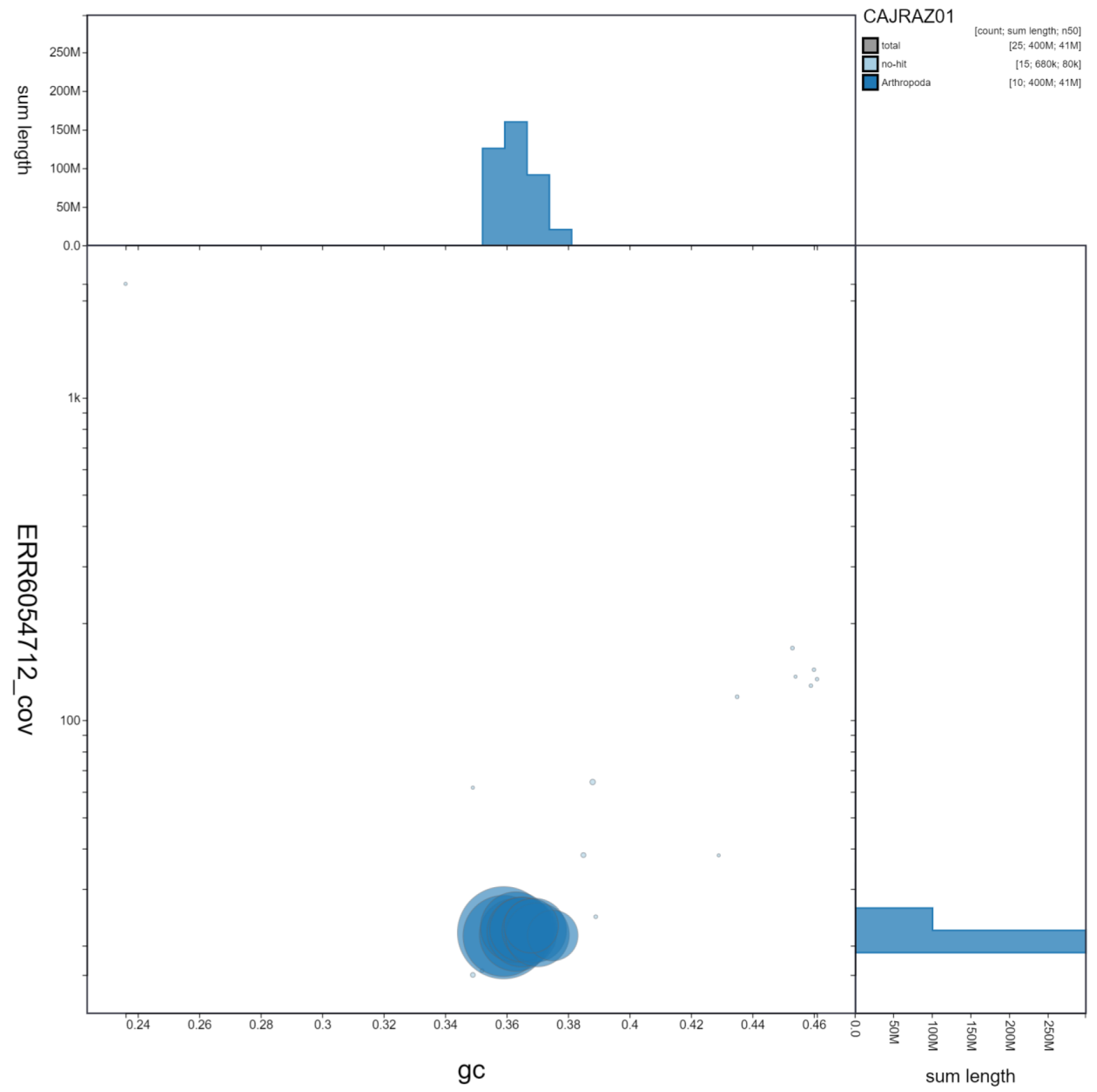

Figure 3. Genome assembly of Coccinella septempunctata, icCocSept1.1: GC coverage. BlobToolKit GC-coverage plot. Scaffolds are coloured by phylum. Circles are sized in proportion to scaffold length Histograms show the distribution of scaffold length sum along each axis. An interactive version of this figure is available at https://blobtoolkit.genomehubs.org/view/icCocSept1.1/dataset/CAJRAZ01/blob.

AMPure PB beads with a $1.8 \mathrm{X}$ ratio of beads to sample to remove the shorter fragments and concentrate the DNA sample. The concentration of the sheared and purified DNA was assessed using a Nanodrop spectrophotometer and Qubit Fluorometer and Qubit dsDNA High Sensitivity Assay kit. Fragment size distribution was evaluated by running the sample on the FemtoPulse system.

\section{Sequencing}

Pacific Biosciences HiFi circular consensus and 10X Genomics read cloud DNA sequencing libraries were constructed according to the manufacturers' instructions. Sequencing was performed by the Scientific Operations core at the Wellcome Sanger Institute on Pacific Biosciences SEQUEL II and Illumina HiSeq $\mathrm{X}$ instruments. Hi-C data were generated using 


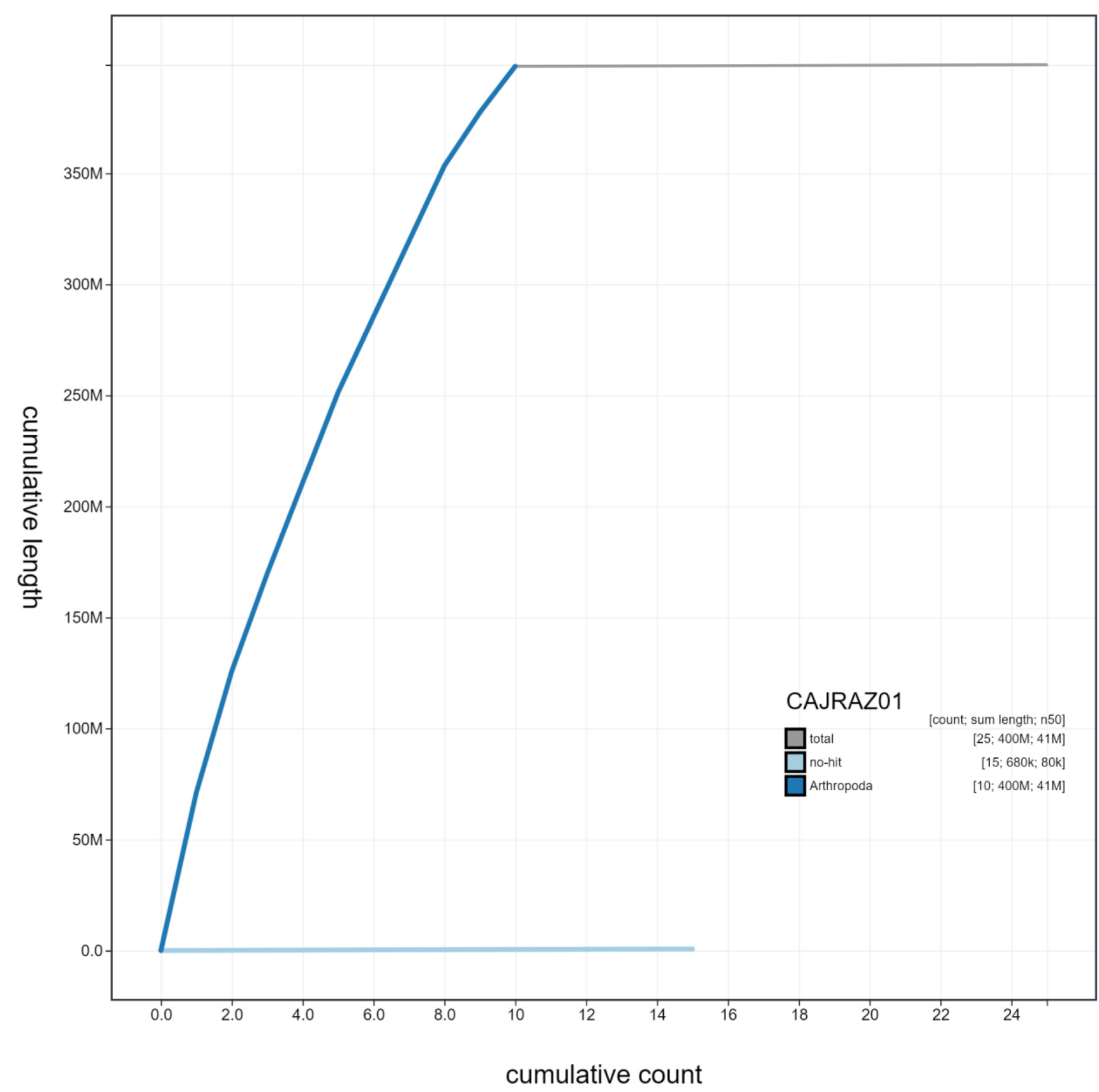

Figure 4. Genome assembly of Coccinella septempunctata, icCocSept1.1: cumulative sequence. BlobToolKit cumulative sequence plot. The grey line shows cumulative length for all scaffolds. Coloured lines show cumulative lengths of scaffolds assigned to each phylum using the buscogenes taxrule. An interactive version of this figure is available at https://blobtoolkit.genomehubs.org/view/icCocSept1.1/ dataset/CAJRAZ01/cumulative.

the Arima v2 Hi-C kit and sequenced on an Illumina NovaSeq 6000 instrument.

\section{Genome assembly}

Assembly was carried out with HiCanu (Nurk et al., 2020); haplotypic duplication was identified and removed with purge_dups
(Guan et al., 2020). One round of polishing was performed by aligning $10 \mathrm{X}$ Genomics read data to the assembly with longranger align, calling variants with freebayes (Garrison \& Marth, 2012). The assembly was then scaffolded with Hi-C data (Rao et al., 2014) using SALSA2 (Ghurye et al., 2019). The assembly was checked for contamination and corrected 


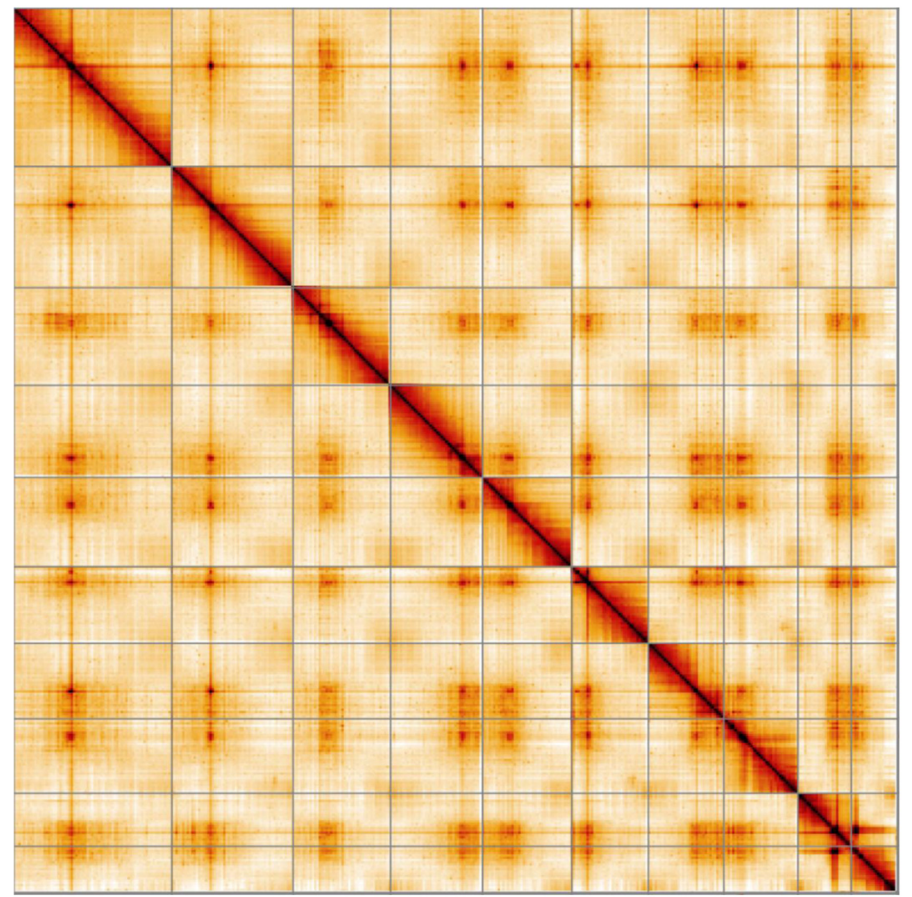

Figure 5. Genome assembly of Coccinella septempunctata, icCocSept1.1: Hi-C contact map. Hi-C contact map of the icCocSept1.1 assembly, visualised in HiGlass.

\section{Table 2. Chromosomal pseudomolecules in the genome assembly of Coccinella septempunctata, icCocSept1.1.}

\begin{tabular}{|c|c|c|c|}
\hline INSDC accession & Chromosome & Size (Mb) & GC\% \\
\hline OU015573.1 & 1 & 71.18 & 35.9 \\
\hline OU015574.1 & 2 & 54.65 & 35.9 \\
\hline OU015575.1 & 3 & 43.94 & 36.3 \\
\hline OU015576.1 & 4 & 41.44 & 36.3 \\
\hline OU015577.1 & 5 & 40.26 & 36.5 \\
\hline OU015578.1 & 6 & 34.46 & 36.5 \\
\hline OU015579.1 & 7 & 34.01 & 36.9 \\
\hline OU015580.1 & 8 & 33.49 & 37 \\
\hline OU015582.1 & 9 & 20.75 & 37.5 \\
\hline OU015581.1 & X & 24.00 & 36.8 \\
\hline OU015583.1 & MT & 0.02 & 23.7 \\
\hline- & Unplaced & 0.66 & 39.7 \\
\hline
\end{tabular}

using the gEVAL system (Chow et al., 2016) as described previously (Howe et al., 2021). Manual curation (Howe et al., 2021) was performed using gEVAL, HiGlass (Kerpedjiev et al., 2018) and Pretext. The mitochondrial genome was assembled using MitoHiFi (Uliano-Silva et al., 2021). The genome was analysed and BUSCO scores generated within the BlobToolKit environment (Challis et al., 2020). Table 3 contains a list of all software tool versions used, where appropriate.
Table 3. Software tools used.

\begin{tabular}{|c|c|c|}
\hline Software tool & Version & Source \\
\hline HiCanu & 2.1 & Nurk et al., 2020 \\
\hline purge_dups & 1.2 .3 & Guan et al., 2020 \\
\hline SALSA2 & 2.2 & Ghurye et al., 2019 \\
\hline longranger align & 2.2 .2 & $\begin{array}{l}\text { https:// } \\
\text { support.10xgenomics. } \\
\text { com/genome-exome/ } \\
\text { software/pipelines/ } \\
\text { latest/advanced/other- } \\
\text { pipelines }\end{array}$ \\
\hline freebayes & 1.3.1-17-gaa2ace8 & Garrison \& Marth, 2012 \\
\hline MitoHiFi & 1.0 & Uliano-Silva et al., 2021 \\
\hline gEVAL & N/A & Chow et al., 2016 \\
\hline HiGlass & 1.11 .6 & Kerpedjiev et al., 2018 \\
\hline PretextView & $0.1 . x$ & $\begin{array}{l}\text { https://github.com/wtsi- } \\
\text { hpag/PretextView }\end{array}$ \\
\hline BlobToolKit & 2.6 .2 & Challis et al., 2020 \\
\hline
\end{tabular}

Ethics/compliance issues

The materials that have contributed to this genome note have been supplied by a Darwin Tree of Life Partner. The submission of materials by a Darwin Tree of Life Partner is subject to the Darwin Tree of Life Project Sampling Code of Practice. By agreeing with and signing up to the Sampling Code of Practice, 
the Darwin Tree of Life Partner agrees they will meet the legal and ethical requirements and standards set out within this document in respect of all samples acquired for, and supplied to, the Darwin Tree of Life Project. Each transfer of samples is further undertaken according to a Research Collaboration Agreement or Material Transfer Agreement entered into by the Darwin Tree of Life Partner, Genome Research Limited (operating as the Wellcome Sanger Institute), and in some circumstances other Darwin Tree of Life collaborators.

\section{Data availability}

European Nucleotide Archive: Coccinella septempunctata (seven-spotted ladybird). Accession number PRJEB44834; https:// identifiers.org/ena.embl/PRJEB44834

The genome sequence is released openly for reuse. The C. septempunctata genome sequencing initiative is part of the Darwin Tree of Life (DToL) project. All raw sequence data and the assembly have been deposited in INSDC databases. The genome will be annotated and presented through the Ensembl pipeline at the European Bioinformatics Institute. Raw data and assembly accession identifiers are reported in Table 1.

\section{Author information}

Members of the University of Oxford and Wytham Woods Genome Acquisition Lab are listed here: https://doi.org/10.5281/ zenodo.4789929.

Members of the Darwin Tree of Life Barcoding collective are listed here: https://doi.org/10.5281/zenodo.4893704.

Members of the Wellcome Sanger Institute Tree of Life programme collective are listed here: https://doi.org/10.5281/ zenodo.5377053.

Members of Wellcome Sanger Institute Scientific Operations: DNA Pipelines collective are listed here: https://doi.org/10.5281/ zenodo.4790456.

Members of the Tree of Life Core Informatics collective are listed here: https://doi.org/10.5281/zenodo.5013542.

Members of the Darwin Tree of Life Consortium are listed here: https://doi.org/10.5281/zenodo.4783559.
Challis R, Richards E, Rajan J, et al.: BlobToolKit--Interactive Quality Assessment of Genome Assemblies. G3 (Bethesda). 2020; 10(4): 1361-74. PubMed Abstract | Publisher Full Text | Free Full Text

Chow W, Brugger K, Caccamo M, et al.: gEVAL - a web-based browser for evaluating genome assemblies. Bioinformatics. 2016; 32(16): 2508-10. PubMed Abstract | Publisher Full Text | Free Full Text Garrison E, Marth G: Haplotype-Based Variant Detection from Short-Read Sequencing. 2012; arXiv: 1207.3907. Reference Source

Ghurye J, Rhie A, Walenz BP, et al.: Integrating Hi-C Links with Assembly Graphs for Chromosome-Scale Assembly. PLoS Comput Biol. 2019; 15(8): e1007273. PubMed Abstract | Publisher Full Text | Free Full Text

Guan D, McCarthy SA, Wood J, et al.: Identifying and Removing Haplotypic Duplication in Primary Genome Assemblies. Bioinformatics. 2020; 36(9): 2896-98.

PubMed Abstract | Publisher Full Text | Free Full Text

Hodek I, Michaud JP, Others: Why Is Coccinella Septempunctata so successful?(A Point-of-View). EJE. 2013; 105(1): 1-12.

Howe K, Chow W, Collins J, et al.: Significantly Improving the Quality of Genome Assemblies through Curation. Gigascience. 2021; 10(1): giaa153. PubMed Abstract | Publisher Full Text | Free Full Text

Kerpedjiev P, Abdennur N, Lekschas F, et al.: HiGlass: Web-Based Visua
Exploration and Analysis of Genome Interaction Maps. Genome Biol. 2018; 19(1): 125.

PubMed Abstract | Publisher Full Text | Free Full Text

Manni M, Berkeley MR, Seppey M, et al.: BUSCo Update: Novel and Streamlined Workflows along with Broader and Deeper Phylogenetic Coverage for Scoring of Eukaryotic, Prokaryotic, and Viral Genomes. Mol Biol Evol. 2021; 38(10): 4647-54.

PubMed Abstract | Publisher Full Text | Free Full Text

Nurk S, Walenz BP, Rhie A, et al.: HiCanu: Accurate Assembly of Segmental Duplications, Satellites, and Allelic Variants from High-Fidelity Long Reads. Genome Res. 2020; 30(9): 1291-1305.

PubMed Abstract | Publisher Full Text | Free Full Text

Rao SSP, Huntley MH, Durand NC, et al:: A 3D Map of the Human Genome at Kilobase Resolution Reveals Principles of Chromatin Looping. Cell. 2014; 159(7): 1665-80.

PubMed Abstract | Publisher Full Text | Free Full Text

Roy H, Brown P: Field Guide to the Ladybirds of Great Britain and Ireland. Bloomsbury Publishing. 2018.

Reference Source

Uliano-Silva M, Nunes JGF, Krasheninnikova $\mathrm{K}$, et al: marcelauliano/MitoHiFi: mitohifi_v2.0. 2021

Publisher Full Text 


\section{Open Peer Review}

\section{Current Peer Review Status:}

\section{Version 1}

Reviewer Report 29 November 2021

https://doi.org/10.21956/wellcomeopenres.19176.r47194

(C) 2021 Park J. This is an open access peer review report distributed under the terms of the Creative Commons Attribution License, which permits unrestricted use, distribution, and reproduction in any medium, provided the original work is properly cited.

\section{Jongsun Park}

InfoBoss Inc, Seoul, South Korea

The manuscript entitled "The genome sequence of the seven-spotted ladybird, Coccinella septempunctata Linnaeus, 1758" describe the whole genome sequence of $C$. septempunctata. All genomic data are properly available in the database and the figures which describe features of the whole genome assembly are properly drawn.

I'm not sure whether the author can also provide gene model of this genome or not because NCBI provides a number of transcripts. I think that just brief information of the gene model of this genome wiould be better for this manuscript.

Is the rationale for creating the dataset(s) clearly described?

Yes

Are the protocols appropriate and is the work technically sound? Yes

Are sufficient details of methods and materials provided to allow replication by others? Yes

Are the datasets clearly presented in a useable and accessible format?

Yes

Competing Interests: No competing interests were disclosed.

Reviewer Expertise: Genomics, Comparative Genomics, Bioinformatics, Biodiversity informatics, Plant Taxonomy

I confirm that I have read this submission and believe that I have an appropriate level of expertise to confirm that it is of an acceptable scientific standard. 
Reviewer Report 25 November 2021

https://doi.org/10.21956/wellcomeopenres.19176.r47192

(c) 2021 Richards S. This is an open access peer review report distributed under the terms of the Creative Commons Attribution License, which permits unrestricted use, distribution, and reproduction in any medium, provided the original work is properly cited.

\section{Stephen Richards}

Human Genome Sequencing Center, Department of Human and Molecular Genetics, Baylor College of Medicine, Houston, TX, USA

The is great genome assembly, of an important species, and I can easily find the data at NCBI and EBI - all of this is good.

My only tiny issue (and it is a tiny tiny issue) is that although the methods are described in the standard manner, and the software versions are used, there could be a better way - perhaps with a document showing example commands and a continuer to download the software - perhaps this needs to be a methods publication for this set of papers that really help a graduate student repeat the assembly?

Otherwise, a wonderful high quality assembly that has made entomology a better place.

Is the rationale for creating the dataset(s) clearly described?

Yes

Are the protocols appropriate and is the work technically sound?

Yes

Are sufficient details of methods and materials provided to allow replication by others? Partly

Are the datasets clearly presented in a useable and accessible format? Yes

Competing Interests: No competing interests were disclosed.

Reviewer Expertise: Arthropod Genomics, Human Genomics, transcriptomics

I confirm that I have read this submission and believe that I have an appropriate level of expertise to confirm that it is of an acceptable scientific standard. 